\title{
Kernos
}

Revue internationale et pluridisciplinaire de religion grecque antique

7| 1994

Varia

Cahiers du GITA, 7 (1992-1993). Les Perses d'Eschyle, textes rassemblés par Paulette GHIRON-BISTAGNE, Alain MOREAU, Jean-Claude TURPIN

\section{Edith Gilis}

\section{OpenEdition}

Journals

Édition électronique

URL : http://journals.openedition.org/kernos/1128

DOI : $10.4000 /$ kernos. 1128

ISSN : 2034-7871

\section{Éditeur}

Centre international d'étude de la religion grecque antique

Édition imprimée

Date de publication : 1 janvier 1994

ISSN : 0776-3824

\section{Référence électronique}

Edith Gilis, «Cahiers du GITA, 7 (1992-1993). Les Perses d'Eschyle, textes rassemblés par Paulette GHIRon-bistagne, Alain moreau, Jean-Claude turpin », Kernos [En ligne], 7 | 1994, mis en ligne le 20 avril 2011, consulté le 21 septembre 2020. URL : http://journals.openedition.org/kernos/1128 ; DOI : https://doi.org/10.4000/kernos. 1128 
particulier, par le biais des reliefs votifs notamment. À cet égard, une incursion vers le domaine cultuel se révèle fort instructive ${ }^{1}$. On sait aussi combien ce motif sera réélaboré, «récupéré» sur le plan funéraire, héroïque, aristocratique, politique, eschatologique... Nombreuses sont, effectivement, les potentialités d'une telle scène, mais il ne faut pas perdre de vue que certaines sont anachroniques. Et, là encore, un dernier point risque de paraître litigieux : l'hypothèse d'un Héraklèsmiroir de la condition humaine, au destin semé de joies, de doutes et de souffrances (dans une optique d'abord aristocratique, puis de plus en plus universelle) est-elle fondamentalement de mise pour les années 530-460 ? Sans doute, mais nous n'en avons guère de preuves, et il faudrait admettre qu'un tel processus d'identification n'en est qu'à ses débuts ${ }^{2}$. Quoi qu'il en soit, je n'affirmerais pas que, dans la première moitié du Ve s., Héraklès, perçu sentimentalement, comble déjà le vide laissé par les grands dieux poliades (p. 192 :...der Moment, wo die Götter in grössere Entfernung rücken).

Ces quelques remarques n'ont d'autre but que d'inciter l'A. à poursuivre une belle recherche menée, on le devine, avec conviction et sur de bonnes bases céramologiques, afin qu'à l'avenir l'interprétation ne s'arrête plus en si bon chemin et puisse s'écarter des sentiers de la vieille tradition mythographique, trop réductrice.

Annie Verbanck-Piérard (Musée Royal de Mariemont)

\section{Notices bibliographiques}

Cahiers du GITA, 7 (1992-1993). 1 vol. 16 x 24 cm, 258 p. (Les Perses d'Eschyle, textes rassemblés par Paulette Ghiron-Bistagne, Alain Moreau, Jean-Claude TuRPIN). ISSN : 0295-9909.

Ce numéro spécial des Cahiers du Gita consacré aux Perses d'Eschyle regroupe dix communications destinées à la fois aux chercheurs et aux étudiants. Le propos est diversifié : certaines études relèvent de la philologie ou de l'histoire de l'art; d'autres s'intéressent aux rapports entre les mythes et la tragédie, ou encore à l'histoire et à la société.

1 Cf. mon article cité supra n. 5 ; j'y publie, p. 103, un fragment inédit d'un skyphos du peintre de Thésée (coll. privée suisse), à ajouter au catalogue de S. Wolf.

2 C. BÉRARD, Étrangler un lion à mains nues, in Images et société en Grèce ancienne, Cahiers d'Archéologie Romande, 36 (1987), p. 177-186. 
Les articles de J. Irigouin, J. Assaël, A. Moreau, L. Bourdaux et J. JouANnA veulent éclairer le texte par une analyse précise, mettre en valeur les aspects essentiels et fournir les matériaux du commentaire. Le premier s'interroge sur la construction métrique et le jeux des sonorités dans le parodos des Perses. J. AssaËL, quant à lui, s'intéresse à la technique de répétition qu' Eschyle emploie avec un art consommé, de manière à produire des effets puissants et variés» (p. 15). Le premier des deux articles d'A. Moreau s'attache à l'examen du vocabulaire et aux difficultés des vers 176-214, portant sur le songe d'Atossa. L. Bourdaux met en évidence l'importance du premier stasimon qui prépare les thèmes essentiels de la tragédie; la puissance conquérante de Xerxès et l'opposition entre ce dernier et le fantôme du «Roi parfait Darios» (p. 71).

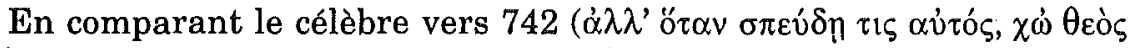
$\left.\sigma v \vee \alpha ́ \pi \tau \varepsilon \tau \alpha_{l}\right)$, avec d'autres textes, dont la prose ionienne des médecins grecs du Ve siècle, J. JouANNA entend prouver que la traduction de cette maxime par P. MAzON doit être modifiée («mais quand un mortel s'emploie à sa perte, les dieux viennent l'aider"). Malgré le désaccord d'une grande partie des critiques J. JouANNA traduit ce vers par : "Mais quand un homme s'active de son côté, la divinité donne aussi un coup de main» en sous-entendant que l'interprétation du verbe $\sigma v v \alpha ́ \pi \tau \varepsilon \sigma \theta \alpha \iota$ signifie : «un dieu sans doute avait touché ses esprits». Cet exposé particulièrement intéressant est repris dans la Revue des Études grecques, 106 (1993), p. 181-194.

La communication très documentée et très précise de S. SAÏD met en relief l'originalité d'Eschyle par rapport aux autres auteurs, notamment lorsque le tragique s'attarde sur l'épisode de Psyttalie. Le poète transforme ainsi ce qui fut d'abord une victoire navale en un double triomphe sur terre et sur mer. Par cette analyse, on peut supposer qu'Eschyle, qui accorde un rôle considérable aussi bien aux hoplites qu'aux marins, annonce la future domination d'Athènes sur de nombreuses cités grecques et étrangères.

Dans une étude très élaborée et passionnante intitulée "Les Perses, la géopolitique et l'histoire", A. TourRaIx est près de faire d'Eschyle le "Père de l'histoire" en lieu et place d'Hérodote. La tragédie est envisagée comme une réflexion sur la politique et sur l'histoire. L'analyse historique est très fine et très détaillée. Le choix d'étudier le rapport entre le souci historique d'Eschyle et son art dramatique est judicieux. Parmi les divers modes d'approche du genre tragique, on ne peut négliger une telle grille d'analyse.

A. Moreau, dans sa communication «La tétralogie des Perses a-telle une unité ?", met également en exergue un type d'analyse important pour saisir correctement tous les aspects d'une tragédie. Il s'intéresse à 
la fois à l'audace de présenter sur scène une réalité contemporaine, au rapport entre tragédie et mythe, et au rôle primordial joué par l'hybris. Les spectateurs qui furent aussi les soldats de Marathon et de Salamine retiennent la leçon donnée par Eschyle; le crime de la démesure est toujours châtié par les dieux.

En observant le «Vase des Perses» du Musée National de Naples, P. Ghiron-Bistagne s'attarde sur deux points qui, selon elle, ont été négligés par la critique : l'étude des gestes "qui jouent un peu le rôle de bulles dans nos bandes dessinées" (p. 151) et une vision plus globale des scènes qui ne s'enchaînent pas obligatoirement de gauche à droite suivant un ordre chronologique.

Ces dix études s'efforcent d'apporter des interprétations nouvelles en variant les points de vue. Le sentiment d'ensemble qui se dégage de la lecture est la diversité, riche et féconde.

Edith GiLis (Université de Liège)

Richard BoDÉÜs, Aristote et la théologie des vivants immortels, QuébecParis, Bellarmin-Les Belles Lettres, 1992. 1 vol. 15 x 22,5 cm, 396 p. (Coll. "Noésis"). ISBN : 2-89007-722-5.

L'A., qui est professeur à l'Université de Montréal, nous avait déjà habitués à des remises en cause assez radicales dans ses savantes études sur Aristote, telles que Le Philosophe et la cité.Recherches sur les rapports entre morale et politique dans la pensée d'Aristote, Paris, 1982. Voici qu'il récidive, si l'on peut dire, en s'en prenant cette fois à l'exégèse traditionnelle de la pensée religieuse du Stagirite. Et, disonsle tout de suite : avec des arguments solides et souvent convaincants.

Le renouvellement de perspective qui est à la base de tout le travail est fondé sur l'idée que, loin d'avoir cherché à élaborer une «théologie naturelle" qui viserait à purifier la notion populaire du divin, Aristote prend appui sur la théologie grecque la plus traditionnelle pour confirmer toute une série de thèses philosophiques. "La métaphysique aristotélicienne n'est pas le moment de l'histoire où la philosophie prétend découvrir la vraie nature des dieux contre l'obscurantisme des traditions grecques, mais, au contraire, le moment où les lumières de la tradition théologique grecque sont supposées éclairer l'obscurité où la philosophie pénètre lorsqu'elle s'efforce de penser les premiers principes» (p. 300). C'est dire notamment qu'en dépit de certaines apparences et de la manière dont on interprète généralement les textes de la Métaphysique et du De Colo qui dissertent du divin, il n'est pas sûr qu'Aristote ait eu là l'intention de trancher scientifiquement des questions de théologie spéculative. 